\title{
Subdiffraction Focusing Enabled by a Fano Resonance
}

\author{
Shuwen Chen, ${ }^{1,2}$ Shilong Jin, ${ }^{1}$ and Reuven Gordon ${ }^{2, *}$ \\ ${ }^{1}$ College of Optoelectronic Science and Engineering, National University of Defense Technology, \\ Changsha 410073, China \\ ${ }^{2}$ Department of Electrical and Computer Engineering, University of Victoria, \\ Victoria, British Columbia, Canada V8W 3V6 \\ (Received 31 March 2014; revised manuscript received 26 June 2014; published 1 August 2014)
}

\begin{abstract}
Radiationless electromagnetic interference (REI) has been used to achieve focusing below Abbe's diffraction limit. Here, we demonstrate an approach to REI that uses the Fano resonance of subwavelength slits to achieve subdiffraction focusing. Two main features of the Fano resonance are critical: (1) The Fano resonance suppresses radiation by destructive interference, thereby allowing for REI, and (2) the Fano resonance creates a resonant field enhancement allowing one to overcome evanescent decay, which is different from past approaches to REI. An analytic theory is introduced to explain these results. While the analytic theory is formulated for a perfect electric conductor, comprehensive numerical simulations show the applicability in the visible regime, where losses and plasmonic effects play a role.
\end{abstract}

DOI: 10.1103/PhysRevX.4.031021

\section{INTRODUCTION}

Because of the decay of evanescent waves, the smallest focus spot of a lens is restricted by Abbe's resolution limit to approximately half the optical wavelength in the medium [1]. To break this diffraction limit, the general approach is to boost the contribution from evanescent waves [2-4]. Since the first proposal to achieve resolution beyond the diffraction limit [5], small apertures or tips were used to confine the beam into a deep subwavelength region [6-10], leading to the development of near-field scanning optical microscopy (NSOM) [11-13]. To relax the requirement of an extremely short working distance that is typical in NSOM, a negative refraction-based perfect lens was proposed [4], in which the evanescent waves are restored by the plasmonic response of a metal slab, producing a subdiffraction image at the other side of the slab. This approach was later demonstrated experimentally $[14,15]$.

Inspired by Fresnel plates, a near-field lens was introduced as an alternative approach to obtain subdiffraction focusing, originating from radiationless electromagnetic interference (REI) [3]. In this approach, a deep-subwavelength-patterned plate creates a rapid transverse oscillation in the near field that suppresses radiation and interferes destructively in the "propagation" direction to produce a narrow central lobe, that is, the focus. This approach was implemented in the microwave regime, with focusing waves emanating from a cylindrical source to an intensity full-width half-maximum

*rgordon@uvic.ca

Published by the American Physical Society under the terms of the Creative Commons Attribution 3.0 License. Further distribution of this work must maintain attribution to the author(s) and the published article's title, journal citation, and DOI.
Subject Areas: Nanophysics, Optics, Plasmonics

(FWHM) of $\lambda / 20$ at a distance of $\lambda / 15$ at $1 \mathrm{GHz}$ [16]. Different structures were proposed for such subwavelength focusing from the microwave $[17,18]$ to the visible regime [19-21], including slot antennas [22], metal-dielectric-metal waveguide arrays [20], and annular slots [17], which were investigated with simulations and analytical formulations [23].

By noting that the basic idea of REI is introducing satellite lobes to destructively interfere with the main lobe and produce a null in the total field near the focus peak, it is interesting to investigate the subdiffraction focusing by exploiting the strong asymmetric Fano resonances that originate from the close coexistence of the destructive coupling between a narrow resonance and a broad resonance (or continuum) [24-26]. Fano resonances have been observed in a number of subwavelength structures in optics, such as nanoparticle and hole arrays, photonic crystals, nanoshells, nanoparticle clusters, and metamaterials [27-34]. The ability of Fano resonance to control the interaction of light with subwavelength structures at the nanoscale is promising in biosensing [35], photovoltaics [36], and cloaking [37]. The Fano resonance line shapes depend on the eigenfrequencies of the narrow and the broad resonances, the exciting efficiencies, and the coupling strength [38]. The hybridization of a dipolar bonding resonance with low energy and an antibonding resonance with high energy exhibits superradiation and subradiation in the extinction spectra $[39,40]$. While the oscillation is in phase for the superradiant pattern, it is out of phase in the subradiant pattern with huge local field enhancement $[28,41]$. The destructive interference is the physical connection to REI that will be explored further in this work.

In this work, we propose an alternative approach to achieve subdiffraction focusing by REI at a Fano 
resonance. We show that closely placed deep subwavelength slits in a metal slab not only exhibit a Fano resonance but also enable subdiffraction focusing around the radiationless regime of that Fano resonance. Furthermore, the field intensity at the focus is boosted by the huge field enhancement of the Fano resonance, which is not typical for REI demonstrations so far. The performance and limits of this kind of subdiffraction focusing are analyzed. Finally, the applicability to real metals in the visible regime is demonstrated by finitedifference time-domain (FDTD) simulations of analogous slit structures.

\section{SUBDIFFRACTION FOCUSING BY THE SLIT-IN-METAL SLAB}

Figure 1 illustrates schematically the subdiffraction focusing produced by evanescent wave interference after a multislit configuration in a metal slab. The perfect electric conductor (PEC) metal slab is infinitely extended in the $x-y$ plane with the thickness comparable to the wavelength. Three deeply subwavelength slits are closely patterned in the metal slab and infinitely extended in the $y$ direction. The focal plane is at a distance $d$ away from the slab. When a transverse magnetic (TM) plane wave passes through the very narrow slits, the interference of both propagating and evanescent waves occurs at the end of the slab, via the diffraction at the discontinuity [42-45]. The interference from different channels (i.e., different modes of the slit array) can be in phase or out of phase, respectively, resulting in enhanced or suppressed radiation. Because of the absence of phase associated with propagation, the evanescent waves should constructively interfere in the $z$ direction. So, the out-of-phase interference of the transverse components produces a nonradiative static field pattern below the diffraction limit.

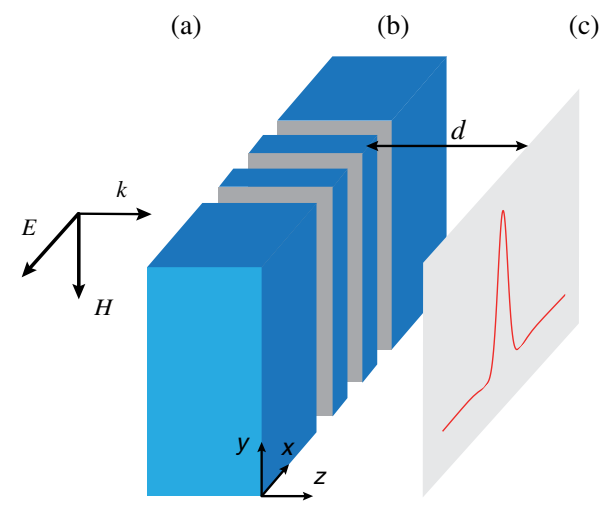

FIG. 1. An illustration of radiationless interference. (a) The transverse magnetic (TM) plane-wave source. (b) Closely placed narrow slits in the metal slab as a plate lens. (c) Focal plane at a distance away from the lens. The inside curve qualitatively shows field amplitude distribution in the direction normal to slits.
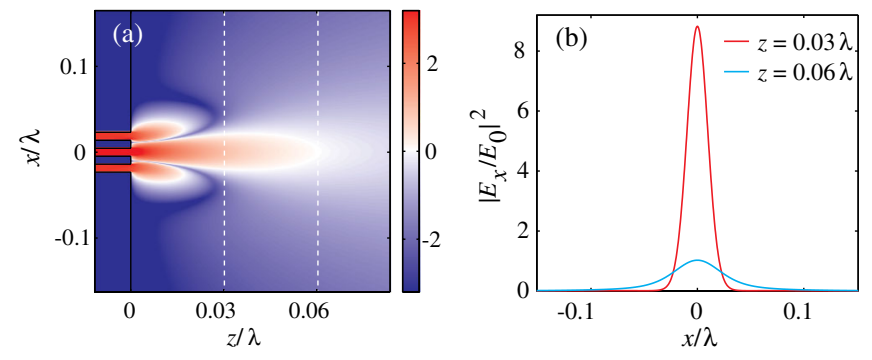

FIG. 2. Subdiffraction focusing. (a) The intensity of the $x$ component of the electric field in log scale in $x-z$ plane. The black solid lines denote the edges of the metal. The white dashed lines indicate the sampling positions. (b) The profile of field intensity at sampling planes normalized to the incident intensity.

Figure 2 shows the subdiffraction focusing effect generated by the multiple-slit system illustrated in Fig. 1. The three slits have the same width, $0.2 \mathrm{~mm}$, separated by $0.2 \mathrm{~mm}$. The thickness of the metal slab is $10 \mathrm{~mm}$. Using comprehensive FDTD electromagnetic simulations, a focusing pattern is observed at wavelength $21.52 \mathrm{~mm}$, which indicates a focus far below the diffraction limit. It is clear that the central lobe interferes destructively with the satellite lobes, resulting in nulls around the main lobe, which plays an essential role in achieving the deep subwavelength focus. The FWHM of the electric field intensity ( $x$-component magnitude squared) is $0.02 \lambda$ and $0.054 \lambda$ at distances of $0.03 \lambda$ and $0.06 \lambda$. An important feature of the subdiffraction focusing is that the fields at the focal plane are significantly stronger than the incident field, which is not typical for REI: Usually, there is an exponential decay of the field at the focus spot $[3,16]$. The Fano resonance in the slit system boosts the field intensity to well above the incident intensity and overcomes the evanescent decay that usually occurs with REI $[19,46]$.

\section{THEORY FOR SUBDIFFRACTION FOCUSING}

REI typically uses the method of backpropagation [3] or spatially shifted beams [22] to find the distribution of the source field. However, distinct from the infinitesimally thin slabs, an additional and important complexity of slabs of finite thickness comes from multiple reflections at the slabair interfaces, which plays an important role in the Fano resonance. The Fano resonance has the dual roles of boosting the near-field intensity to provide evanescent wave amplification and of providing the interference to suppress radiation, which is a requirement of REI. Here, we use a mode-matching-based theory to analyze these features of the subdiffraction focusing.

For a TM plane wave, the main nonzero field components are $E_{x}$ and $H_{y}$. The $P$ eigenmodes $E_{x}^{p}$ in the $N$ slits with width $a_{n}$ located at $x_{n}$ respectively, can be written as

$$
E_{x}^{p}(x)=\sum_{n=1}^{N} \kappa_{n} \operatorname{rect}\left[\left(x+x_{n}\right) / a_{n}\right],
$$


where $\operatorname{rect}(\cdot)$ is the rectangular function. The mode coefficients $\kappa_{n}$ can be found by applying the orthogonality of the modes, $\int_{-\infty}^{\infty} E_{x}^{p}(x) H_{y}^{q}(x) d x=\delta_{p q}$, where $\delta_{p q}$ is the Kronecker delta function. The contributions of different modes to propagating electromagnetic waves and the total transmission can be found by applying the continuity of the tangential components of the electric and magnetic fields at the boundary of slab-air interfaces. This is similar to past single-mode matching approaches [44], but here at least two modes are retained and the coupling between the modes is critical to the resulting physics, as will be discussed below. Details of the formulation are provided in the Appendix.

Figure 3 shows the analytical result that the Fano resonance originates from the weak coupling between the modes in three identical slits with width $a$, located at $-2 a, 0$, and $2 a$. Following Eq. (1), the even modes can be found as $E_{1}=\operatorname{rect}[(x+2 a) / a] / 2+$ $\operatorname{rect}(x / a) / \sqrt{2}+\operatorname{rect}[(x-2 a) / a] / 2$ (mode 1$)$ and $E_{3}=$ $\operatorname{rect}[(x+2 a) / a] / 2-\operatorname{rect}(x / a) / \sqrt{2}+\operatorname{rect}[(x-2 a) / a] / 2$ (mode 3). By symmetry, the odd modes play no role. Mode 1 and mode 3 are, respectively, broad and narrow in the transmission spectra. In Fig. 3, it is assumed that the thickness of metal is unity, and the slit width $a=0.02$. The transmission is normalized to the single-channel limit [47], and we have investigated the surpassing of this singlechannel limit elsewhere [48]. It is clear that the mode analysis produces a Fano line shape. The narrow resonance is at the high-energy side of the broad one (i.e., left side in Fig. 3). The interference at the high-energy side of the

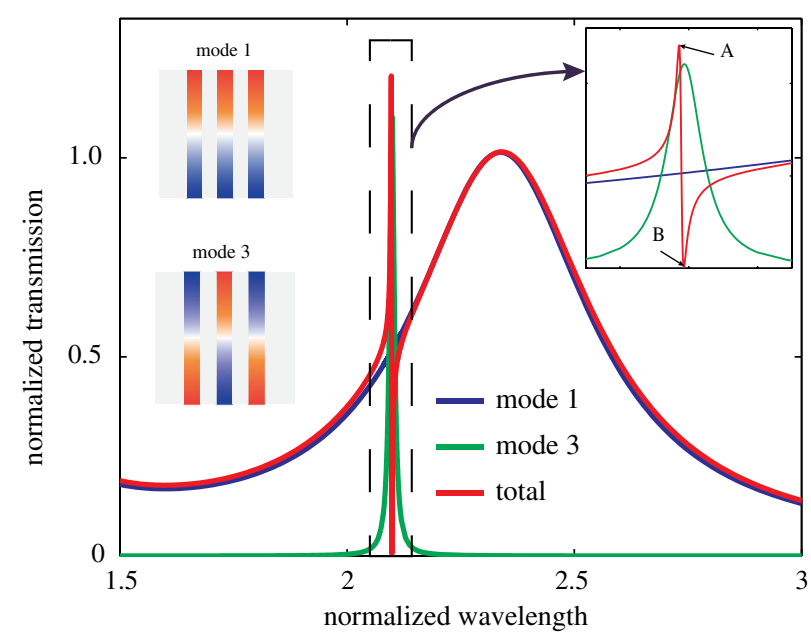

FIG. 3. Modal analysis of Fano resonance in three slits. The low-energy mode 1 is dipolar and spectrally broad, resulting from in-phase interference among slit dipoles. The high-energy mode 3 is hexpolelike and spectrally narrow, resulting from out-of-phase interference among slit dipoles. The coupling of these modes yields asymmetric Fano resonance. The wavelength is normalized by the thickness of the slab, and the transmission is normalized by the single-channel limit. The inset is a zoomed-in view of the coupling. narrow resonance is in phase, which enhances the radiation, resulting in superradiative behavior. Since a $\pi$-phase jump happens around the resonance, the interference at the lowenergy side is out of phase [26], which suppresses the radiation, resulting in subradiative behavior. For the out-ofphase interference, a pattern source rapidly oscillating in the transverse direction will be generated, which is associated with subdiffraction focusing. Thereby, the connection of subdiffraction focusing, that is, REI, to Fano resonance is established with this mode-analysis method.

The position of the Fano resonance is mainly dependent on the narrow resonance of the $E_{x}^{3}$ mode, which is determined by $\beta h+\Delta \Phi_{r}=\pi$, where $\Delta \Phi_{r}$ is the shift caused by the reflection $r$ at the interface between air and slab, and $r$ can be found by Eq. (A10). In our configuration, as related to Fig. 3 , the integral $I$ can be found, which gives $\Delta \Phi_{r}=2.44 \pi a$. From this result, the position of the Fano resonance is $\lambda_{\text {Fano }}=2 h+2.44 w$, where $h$ and $w$ are the physical thickness of the slab and width of the slits.

Figure 4 shows a fit of the transmission profile to a Fano resonance modulated by a Lorentzian resonance, i.e.,

$$
T_{\text {fit }}=C \frac{(\zeta+q)^{2}}{\zeta^{2}+1} \cdot \frac{\gamma_{L}^{2}}{\left(\lambda-\lambda_{L}\right)^{2}+\gamma_{L}^{2}},
$$

where $\zeta=\left(\omega-\omega_{F}\right) / \gamma_{F}$ and $C$ is a normalized parameter. From this fit, we find that the asymmetry parameter is approximately unity, and the Fano resonance width is $0.03 \%$ of the resonance frequency. We have also repeated this procedure for several slit widths $a$ ranging from 0.005 to 0.1 , and we have found that the asymmetry parameter is always around unity and narrower slits give narrower resonance because of mode shape mismatch, with the resonance width taking on the range from $0.02 \%$ to $0.04 \%$.

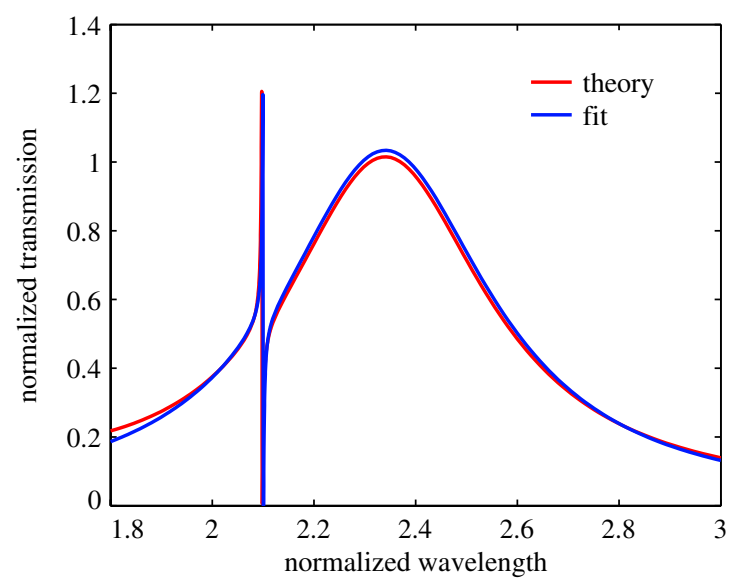

FIG. 4. Fitting the transmission to a Fano resonance. The dimensions are the same as those in Fig. 3; the model parameters are $\lambda_{F}=2.1, \lambda_{L}=2.34, \gamma_{F}=0.0003 \omega_{F}$, and $\gamma_{L}=0.2516$ and $q=1.1$. 


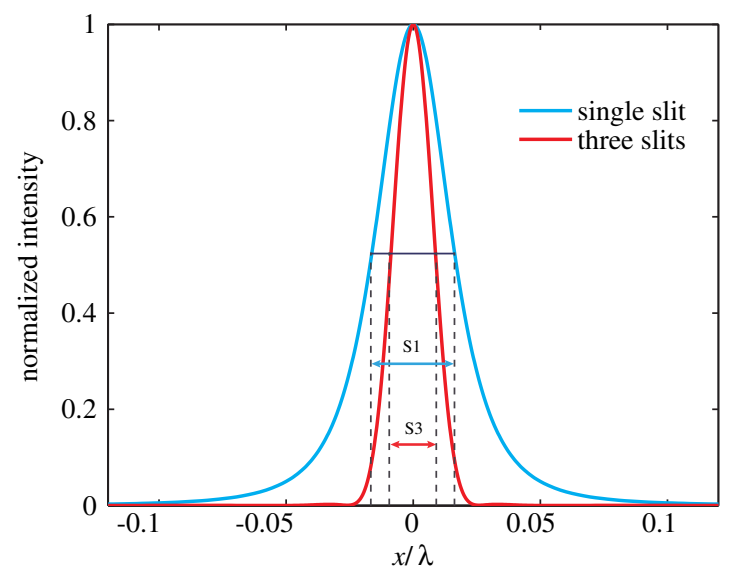

FIG. 5. Performance of Fano REI compared with a single slit. The dimensions are the same as those in Fig. 2, and the focal length is $z=0.03 \lambda$. The intensity is normalized to its maximum value for each plate. The focal sizes of three slits and a single slit are $S 3=0.02 \lambda$ and $S 1=0.036 \lambda$, respectively.

Figure 5 shows the performance of REI enabled by a Fano resonance. We can evaluate the performance of Fano REI seen for the three-slit system by considering the ratio between the focal length and the spot size, as proposed previously [49]. From this, we find the ratio to be 1.5 , which is larger than the single slit by a factor of 1.8 , as shown in Fig. 5.

\section{SCALING TO THE VISIBLE REGIME}

It is important to scale from low frequencies to the visible regime for real metals with finite permittivity where loss and plasmonic features come into play [50].

Figure 6 shows subdiffraction focusing in the visible regime using FDTD simulations with an experimental dielectric constant of silver [51]. Figure 6(a) shows the intensity distribution of subdiffraction focusing at the wavelength $680 \mathrm{~nm}$. The thickness of the silver film is chosen to be on resonance for both three slits and a single slit, that is, $100 \mathrm{~nm}$ and $142 \mathrm{~nm}$. Figure 6(b) shows a comparison of three-slit Fano REI and the single slit. The
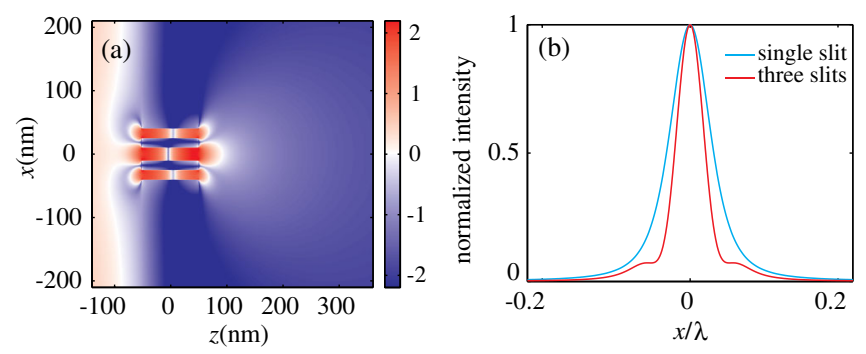

FIG. 6. Subdiffraction focusing in the visible regime. (a) The intensity of the $x$ component of the electric field on a log scale in the $x-z$ plane. The central slit is $20 \mathrm{~nm}$ in width, and satellite slits are $15 \mathrm{~nm}$, separated by $15 \mathrm{~nm}$. (b) The profile of the field intensity $32 \mathrm{~nm}$ away from the slits, compared with a single slit. field distribution and focusing property are similar to that of PEC slabs. This similarity is due to the inherent scalability of the Fano resonance in the slit system.

\section{DISCUSSIONS}

Applying the superfocusing metric [49], that is, the ratio of the focal length and the focal size, we can compare this Fano subdiffraction focusing with the other approaches. We find that the poor man's perfect lens is 0.5 in the UV $[4,49]$, the typical REI is $1.34[3,22,49]$, and the Fano subdiffraction focusing in this work is 1.5 at the microwave regime and 0.6 in the visible regime. Therefore, the Fano scheme is comparable to past REI demonstrations but has the additional advantage of boosting the local field intensity.

In the microwave and $\mathrm{THz}$ regimes, metals are well approximated as perfect conductors, and the coupling between the narrow mode and the broad mode will produce a sharp Fano resonance; therefore, a deep subdiffraction focus should be well defined. In the visible and nearinfrared regime, because of metal loss, the transmission resonances of individual modes (especially the narrow mode) will become less sharp. To demonstrate the influence of losses, we have included a realistic calculation showing less-defined focusing in Fig. 6. The available nanofabrication technology also places a limit on the size of the slit; however, slits as small as $1 \mathrm{~nm}$ have been fabricated reliably [52]. We do not study the influence of these tolerances here.

The proposed structure dimensions for the visible light are compatible with the present nanofabrication achievements. This is based on the recent experimental demonstrations; for example, a 14-nm slit in the length of $3 \mu \mathrm{m}$ was made in a metal-insulator-metal (MIM) waveguide [53], and even a 1-nm slit has recently been achieved by atomic-layer deposition [52]. One of the advantages of the approach introduced in this work to implement REI at the visible regime is that, unlike the dielectrically loaded MIM waveguide [20,54], the proposed structure relaxes the requirement of a varying refractive index in the slits. In addition, the Fano superfocusing can be directly excited by a plane wave, which avoids the challenge of exciting the highest mode of MIM-waveguide arrays as required in our past work [20].

This approach can be generalized to more than two modes. For example, there are three modes in the five-slit configuration, and the phase distributions are found as " +++++ ," " $-0+0-$," and ".+-+-+ ." The couplings between these modes produce two Fano resonances, and REI can be found around the resonance of the highestorder mode where destructive interference is obtained between adjacent lobes. Because of the complexity in effectively designing the coupling of multiple modes, we typically found that the focus is less confined than in the three-slit one. Additionally, by symmetry, three slits is the 
minimum required to obtain subdiffraction focusing by a Fano resonance for normal incidence.

\section{CONCLUSIONS}

In conclusion, we have demonstrated that the Fano resonance of the multiple-slit system can produce REI to achieve subdiffraction focusing. This Fano resonance allows for suppressing radiation through destructive interference, which is critical to the physics of REI. It also resonantly enhances the local field from the nonradiating (evanescent) components, which gives a "brighter" focus spot. While the theoretical formulation was developed for PECs, we showed that the concepts can be extended into the visible regime. This is of interest to many applications of near-field imaging and spectroscopy, where it is critical to have a noninvasive probe that operates at a distance, for example, subdiffraction imaging inside cells [55] or imaging defects inside semiconductors [56].

\section{ACKNOWLEDGMENTS}

The authors acknowledge funding from the NSERC Strategic Network Grant Program.

\section{APPENDIX: MODE MATCHING FOR THREE SLITS}

Considering three identical slits with width $a$, located at $-d, 0, d$, the even modes can be found as

$$
E_{x}^{1}(x)=\frac{1}{2} \mathrm{r}\left(\frac{x+d}{a}\right)+\frac{1}{\sqrt{2}} \mathrm{r}\left(\frac{x}{a}\right)+\frac{1}{2} \mathrm{r}\left(\frac{x-d}{a}\right)
$$

and

$$
E_{x}^{3}(x)=\frac{1}{2} \mathrm{r}\left(\frac{x+d}{a}\right)-\frac{1}{\sqrt{2}} \mathrm{r}\left(\frac{x}{a}\right)+\frac{1}{2} \mathrm{r}\left(\frac{x-d}{a}\right),
$$

where $r(\cdot)$ is the rectangular function. When a TM plane wave impinges on the triple-slit system, the reflected magnetic fields can be written as the expansion of plane-wave modes, both propagating and evanescent. Considering mode $E_{x}^{1}(x)$ only, and applying the continuity of the $E_{x}$ and $H_{y}$ at the incident interface, we get

$$
1+\int_{-\infty}^{\infty} \frac{k_{z}\left(k_{x}\right)}{k_{0}} s\left(k_{x}\right) e^{i k_{x} x} d k_{x}=t E_{x}^{1}(x)
$$

and

$$
\frac{1}{Z_{0}}\left[1-\int_{-\infty}^{\infty} s\left(k_{x}\right) e^{i k_{x} x} d k_{x}\right]=t H_{y}^{1}(x),
$$

where $k_{z}\left(k_{x}\right)=\sqrt{k_{0}^{2}-k_{x}^{2}}, s\left(k_{x}\right)$ is the reflection amplitude, and $t$ is the transmission coefficient. The dependence of fields on $x$ is ignored in the following for simplicity. Taking the Fourier transform of Eq. (A1) gives

$$
s\left(-k_{x}\right)=-\frac{k_{0}}{k_{z}} \delta\left(k_{x}\right)+\frac{t k_{0}}{2 \pi k_{z}} \tilde{F}\left(E_{x}^{1}\right),
$$

where $\tilde{F}\left(E_{x}^{1}\right)=\int_{-\infty}^{\infty} E_{x}^{1}(x) e^{-i k_{x} x} d x$. Incorporating Eq. (A3) into Eq. (A2) gives

$$
2-\int_{-\infty}^{\infty} \frac{k_{0} t}{2 \pi k_{z}} \tilde{F}\left(E_{x}^{1}\right) e^{-i k_{x} x} d k_{x}=Z_{0} t H_{y}^{1}
$$

Multiplying Eq. (A4) by $E_{x}^{1}(x)$ and integrating over $x$ gives

$$
2 \int_{-\infty}^{\infty} E_{x}^{1} d x-\int_{-\infty}^{\infty} \frac{t k_{0}}{2 \pi k_{z}}\left[\tilde{F}\left(E_{x}^{1}\right)\right]^{2} d k_{x}=Z_{0} t \int_{-\infty}^{\infty} H_{y}^{1} E_{x}^{1} d x
$$

The transmission coefficient $t$ is found as

$$
t=\frac{2 \int_{-\infty}^{\infty} E_{x}^{1} d x}{\int_{-\infty}^{\infty} \frac{k_{0}}{2 \pi k_{z}}\left[\tilde{F}\left(E_{x}^{1}\right)\right]^{2} d k_{x}+Z_{0} \int_{-\infty}^{\infty} H_{y}^{1} E_{x}^{1} d x} .
$$

Similarly, on the other side of the slab, that is, the exit interface, we find that

$$
(1+r) E_{x}^{1}(x)=\int_{-\infty}^{\infty} \frac{k_{z}\left(k_{x}\right)}{k_{0}} g\left(k_{x}\right) e^{i k_{x} x} d k_{x}
$$

and

$$
(1-r) H_{y}^{1}(x)=\frac{1}{Z_{0}} \int_{-\infty}^{\infty} g\left(k_{x}\right) e^{i k_{x} x} d k_{x}
$$

The Fourier transform of Eq. (A6) yields

$$
g\left(k_{x}\right)=\frac{1+r}{2 \pi} \frac{k_{0}}{k_{z}} \tilde{F}\left[E_{x}^{1}(x)\right]
$$

Inserting Eq. (A8) into Eq. (A7) leads to

$$
(1-r) H_{y}^{1}(x)=\frac{1+r}{2 \pi Z_{0}} \int_{-\infty}^{\infty} \frac{k_{0}}{k_{z}} \tilde{F}\left[E_{x}^{1}(x)\right] e^{i k_{x} x} d k_{x}
$$

Multiplying Eq. (A9) by $E_{x}^{1}(x, h)$ and integrating over $x$ yields

$$
\frac{1-r}{1+r}=\frac{\int_{-\infty}^{\infty} \frac{k_{0}}{k_{z}}\left[\tilde{F}\left(E_{x}^{1}\right)\right]^{2} d k_{x}}{2 \pi Z_{0} \int_{-\infty}^{\infty} H_{y}^{1}(x) E_{x}^{1}(x) d x} .
$$


Finally, the transmission $T_{1}$ related to the mode $E_{x}^{1}(x)$ is given by

$$
T_{1}=\left|\frac{\sqrt{1-|r|^{2}} \exp (i \beta h)}{1-r^{2} \exp (2 i \beta h)}\right|^{2} \int_{-\infty}^{\infty}\left[t H_{y}^{1}\right]^{*} t E_{x}^{1} d x
$$

where $h$ is the thickness of the metal slab and $\beta$ is the propagation constant in slits.

The same procedures can be applied to the mode $E_{x}^{3}(x)$, and the related transmission $T_{3}$ is found as

$$
T_{3}=\left|\frac{\sqrt{1-\left|r_{3}\right|^{2}} \exp \left(i \beta_{3} h\right)}{1-r_{3}^{2} \exp \left(2 i \beta_{3} h\right)}\right|^{2} \int_{-\infty}^{\infty}\left[t_{3} H_{y}^{3}\right]^{*} t_{3} E_{x}^{3} d x
$$

Next, we will discuss the total transmission, including both mode 1 and mode 3 . Considering the cross coupling between the two modes, the following equations are found by applying the continuity of the tangential components of the electric and magnetic fields at the boundary of $z=0$ and $z=h$, respectively:

$$
\begin{aligned}
1+\int_{-\infty}^{\infty} \frac{k_{z}}{k_{0}} s\left(k_{x}\right) e^{i k_{x} x} d k_{x} & =c_{1}^{+} E_{1}^{+}+c_{1}^{-} E_{1}^{-}+c_{3}^{+} E_{3}^{+}+c_{3}^{-} E_{3}^{-}, \\
1-\int_{-\infty}^{\infty} s\left(k_{x}\right) e^{i k_{x} x} d k_{x} & =c_{1}^{+} E_{1}^{+}-c_{1}^{-} E_{1}^{-}+c_{3}^{+} E_{3}^{+}-c_{3}^{-} E_{3}^{-}, \\
\int_{-\infty}^{\infty} \frac{k_{z}}{k_{0}} g\left(k_{x}\right) e^{i k_{x} x} d k_{x} & =c_{1}^{+} E_{1}^{+}+c_{1}^{-} E_{1}^{-}+c_{3}^{+} E_{3}^{+}+c_{3}^{-} E_{3}^{-}, \\
\int_{-\infty}^{\infty} g\left(k_{x}\right) e^{i k_{x} x} d k_{x} & =c_{1}^{+} E_{1}^{+}-c_{1}^{-} E_{1}^{-}+c_{3}^{+} E_{3}^{+}-c_{3}^{-} E_{3}^{-},
\end{aligned}
$$

where $s\left(k_{x}\right)$ and $g\left(k_{x}\right)$ are the field amplitudes of a plane wave with the $x$ component of the wave vector $k_{x}, E_{1}^{+}$and $E_{3}^{+}$are the forward mode 1 and mode $3, E_{1}^{-}$and $E_{3}^{-}$are the backward ones, and $c_{1}^{+}, c_{1}^{-}, c_{3}^{+}$, and $c_{3}^{-}$are unknown coupling coefficients. By applying similar procedures as in the single-mode matching, also using the orthogonality, the coefficient vector $\mathbf{X}$ is obtained as

$$
\mathbf{X}=\mathbf{A}^{-1} \mathbf{b}
$$

with

$$
\mathbf{A}=\left[\begin{array}{cccc}
I_{11}+a & I_{11}-a & I_{13} & I_{13} \\
I_{13} & I_{13} & I_{33}+a & I_{33}-a \\
e^{2 j \beta_{1} h}\left(I_{11}-a\right) & I_{11}+a & e^{j\left(\beta_{1}+\beta_{3}\right) h} I_{13} & e^{j\left(\beta_{1}-\beta_{3}\right) h} I_{13} \\
e^{j\left(\beta_{1}+\beta_{3}\right) h} I_{13} & e^{j\left(\beta_{1}-\beta_{3}\right) h} I_{13} & e^{2 j \beta_{3} h}\left(I_{33}-a\right) & I_{33}+a
\end{array}\right], \quad \mathbf{b}=\left[\begin{array}{c}
2 a(1+1 / \sqrt{2}) \\
2 a(1-1 / \sqrt{2}) \\
0 \\
0
\end{array}\right]
$$

where $\beta_{1}$ and $\beta_{3}$ are the propagation constants of modes, and

$$
\begin{aligned}
& I_{11}=\int_{-\infty}^{\infty} \frac{\sin ^{2}(\pi a u)}{\pi^{2} u^{2} \sqrt{1-u^{2}}}\left[\cos (2 \pi d u)+\frac{1}{\sqrt{2}}\right]^{2} d u \\
& I_{13}=\int_{-\infty}^{\infty} \frac{\sin ^{2}(\pi a u)}{\pi^{2} u^{2} \sqrt{1-u^{2}}}\left[\cos ^{2}(2 \pi d u)-\frac{1}{2}\right] d u, \\
& I_{33}=\int_{-\infty}^{\infty} \frac{\sin ^{2}(\pi a u)}{\pi^{2} u^{2} \sqrt{1-u^{2}}}\left[\cos (2 \pi d u)-\frac{1}{\sqrt{2}}\right]^{2} d u
\end{aligned}
$$

The transmitted power is calculated by using the Poynting theorem, and the total transmission cross section is found as

$$
\sigma_{\mathrm{T}}=a\left(\left|c_{1}^{+}\right|^{2}-\left|c_{1}^{-}\right|^{2}+\left|c_{3}^{+}\right|^{2}-\left|c_{3}^{-}\right|^{2}\right)
$$

[1] E. Abbe, Beiträge zur Theorie des Mikroskops und der Mikroskopischen Wahrnehmung, Arch. F. Microsc. Anat. 9, 413 (1873).

[2] D. Lu and Z. Liu, Hyperlenses and Metalenses for Far-Field Super-Resolution Imaging, Nat. Commun. 3, 1205 (2012).

[3] R. Merlin, Radiationless Electromagnetic Interference: Evanescent-Field Lenses and Perfect Focusing, Science 317, 927 (2007).

[4] J. B. Pendry, Negative Refraction Makes a Perfect Lens, Phys. Rev. Lett. 85, 3966 (2000).

[5] E. H. Synge, A Suggested Method for Extending Microscopic Resolution into the Ultra-microscopic Region, Philos. Mag. 6, 356 (1928).

[6] D. K. Gramotnev and S. I. Bozhevolnyi, Nanofocusing of Electromagnetic Radiation, Nat. Photonics 8, 13 (2014).

[7] D. K. Gramotnev and S. I. Bozhevolnyi, Plasmonics Beyond the Diffraction Limit, Nat. Photonics 4, 83 (2010). 
[8] E. Betzig and J. K. Trautman, Near-Field Optics: Microscopy, Spectroscopy, and Surface Modification Beyond the Diffraction Limit, Science 257, 189 (1992).

[9] E. Betzig, J. K. Trautman, T. D. Harris, J. S. Weiner, and R. L Kostelak, Breaking the Diffraction Barrier: Optical Microscopy on a Nanometric Scale, Science 251, 1468 (1991).

[10] E. A. Ash and G. Nicholls, Super-resolution Aperture Scanning Microscope, Nature (London) 237, 510 (1972).

[11] D. Richards, Nano-Optics and Near-Field Optical Microscopy (Artech House, Norwood, MA, 2009).

[12] J. H. Kim and K. B. Song, Recent Progress of Nanotechnology with NSOM, Micron 38, 409 (2007).

[13] T. Kalkbrenner, U. Hakanson, A. Schädle, S. Burger, C. Henkel, and V. Sandoghdar, Optical Microscopy via Spectral Modifications of a Nanoantenna, Phys. Rev. Lett. 95, 200801 (2005).

[14] Z. Liu, H. Lee, Y. Xiong, C. Sun, and X. Zhang, Far-Field Optical Hyperlens Magnifying Sub-Diffraction-Limited Objects, Science 315, 1686 (2007).

[15] N. Fang, Sub-Diffraction-Limited Optical Imaging with a Silver Superlens, Science 308, 534 (2005).

[16] A. Grbic and R. Merlin, Near-Field Focusing Plates and Their Design, IEEE Trans. Antennas Propag. 56, 3159 (2008).

[17] M. F. Imani and A. Grbic, Planar Near-Field Plates, IEEE Trans. Antennas Propag. 61, 5425 (2013).

[18] A. Grbic, R. Merlin, E. M. Thomas, and M. F. Imani, NearField Plates: Metamaterial Surfaces/Arrays for Subwavelength Focusing and Probing, Proc. IEEE 99, 1806 (2011).

[19] V. Intaraprasonk, Z. Yu, and S. Fan, Combining Radiationless Interference with Evanescent Field Amplification, Opt. Lett. 35, 1659 (2010).

[20] R. Gordon, Proposal for Superfocusing at Visible Wavelengths Using Radiationless Interference of a Plasmonic Array, Phys. Rev. Lett. 102, 207402 (2009).

[21] Y. Wang, A. M. Wong, L. Markley, A. S. Helmy, and G. V. Eleftheriades, Plasmonic Meta-screen for Alleviating the Trade-Offs in the Near-Field Optics, Opt. Express 17, 12351 (2009).

[22] L. Markley, A. M. H. Wong, Y. Wang, and G. V. Eleftheriades, Spatially Shifted Beam Approach to Subwavelength Focusing, Phys. Rev. Lett. 101, 113901 (2008).

[23] M. F. Imani and A. Grbic, An Analytical Investigation of Near-Field Plates, Metamaterials 4, 104 (2010).

[24] C. Ott, A. Kaldun, P. Raith, K. Meyer, M. Laux, J. Evers, C. H. Keitel, C. H. Greene, and T. Pfeifer, Lorentz Meets Fano in Spectral Line Shapes: A Universal Phase and Its Laser Control, Science 340, 716 (2013).

[25] B. Luk'yanchuk, N. I. Zheludev, S. A. Maier, N. J. Halas, P. Nordlander, H. Giessen, and C. T. Chong, The Fano Resonance in Plasmonic Nanostructures and Metamaterials, Nat. Mater. 9, 707 (2010).

[26] A. E. Miroshnichenko, S. Flach, and Y. S. Kivshar, Fano Resonances in Nanoscale Structures, Rev. Mod. Phys. 82, 2257 (2010).

[27] V. Grigoriev, S. Varault, G. Boudarham, B. Stout, J. Wenger, and N. Bonod, Singular Analysis of Fano Resonances in Plasmonic Nanostructures, Phys. Rev. A 88, 063805 (2013).
[28] T. J. Arruda, A. S. Martinez, and F. A. Pinheiro, Unconventional Fano Effect and Off-Resonance Field Enhancement in Plasmonic Coated Spheres, Phys. Rev. A 87, 043841 (2013).

[29] S. H. Mousavi, A. B. Khanikaev, and G. Shvets, Optical Properties of Fano-Resonant Metallic Metasurfaces on a Substrate, Phys. Rev. B 85, 155429 (2012).

[30] U. Naether and M. I. Molina, Fano Resonances in Magnetic Metamaterials, Phys. Rev. A 84, 043808 (2011).

[31] V. A. Fedotov, N. Papasimakis, E. Plum, A. Bitzer, M. Walther, P. Kuo, D. P. Tsai, and N. I. Zheludev, Spectral Collapse in Ensembles of Metamolecules, Phys. Rev. Lett. 104, 223901 (2010).

[32] B. Auguíe and W. L. Barnes, Collective Resonances in Gold Nanoparticle Arrays, Phys. Rev. Lett. 101, 143902 (2008).

[33] M. Sarrazin, J.-P. Vigneron, and J.-M. Vigoureux, Role of Wood Anomalies in Optical Properties of Thin Metallic Films with a Bidimensional Array of Subwavelength Holes, Phys. Rev. B 67, 085415 (2003).

[34] S. Fan and J. D. Joannopoulos, Analysis of Guided Resonances in Photonic Crystal Slabs, Phys. Rev. B 65, 235112 (2002).

[35] C. Wu, A. B. Khanikaev, R. Adato, N. Arju, A. A. Yanik, H. Altug, and G. Shvets, Fano-Resonant Asymmetric Metamaterials for Ultrasensitive Spectroscopy and Identification of Molecular Monolayers, Nat. Mater. 11, 69 (2012).

[36] H. A. Atwater and A. Polman, Plasmonics for Improved Photovoltaic Devices, Nat. Mater. 9, 205 (2010).

[37] C. Argyropoulos, P. Y. Chen, F. Monticone, and G. D'Aguanno, Nonlinear Plasmonic Cloaks to Realize Giant All-Optical Scattering Switching, Phys. Rev. Lett. 108, 263905 (2012).

[38] V. Giannini, Y. Francescato, H. Amrania, C. C. Phillips, and S. A. Maie, Fano Resonances in Nanoscale Plasmonic Systems: A Parameter-Free Modeling Approach, Nano Lett. 11, 2835 (2011).

[39] A. Christ, Y. Ekinci, H. H. Solak, N. A. Gippius, S. G. Tikhodeev, and O. J. F. Martin, Controlling the Fano Interference in a Plasmonic Lattice, Phys. Rev. B 76, 201405 (2007).

[40] E. Prodan, C. Radloff, N. J. Halas, and P. Nordlander, A Hybridization Model for the Plasmon Response of Complex Nanostructures, Science 302, 419 (2003).

[41] J. Wang, X. Liu, L. Li, J. He, C. Fan, Y. Tian, P. Ding, D. Chen, Q. Xue, and E. Liang, Huge Electric Field Enhancement and Highly Sensitive Sensing Based on the Fano Resonance Effect in an Asymmetric Nanorod Pair, J. Opt. 15, 105003 (2013).

[42] P. Lalanne, J. P. Hugonin, and J. C. Rodier, Theory of Surface Plasmon Generation at Nanoslit Apertures, Phys. Rev. Lett. 95, 263902 (2005).

[43] R. Gordon, Vectorial Method for Calculating the Fresnel Reflection of Surface Plasmon Polaritons, Phys. Rev. B 74, 153417 (2006).

[44] R. Gordon, Near-Field Interference in a Subwavelength Double Slit in a Perfect Conductor, J. Opt. A: Pure Appl. Opt. 8, L1 (2006).

[45] R. Gordon, Light in a Subwavelength Slit in a Metal: Propagation and Reflection, Phys. Rev. B 73, 153405 (2006). 
[46] F. J. Garciá-Vidal, H. J. Lezec, T. W. Ebbesen, and L. Martin-Moreno, Multiple Paths to Enhance Optical Transmission through a Single Subwavelength Slit, Phys. Rev. Lett. 90, 213901 (2003).

[47] Z. Ruan and S. Fan, Superscattering of Light from Subwavelength Nanostructures, Phys. Rev. Lett. 105, 013901 (2010).

[48] S. Chen, S. Jin, and R. Gordon, Super-Transmission from a Finite Subwavelength Arrangement of Slits in a Metal Film, Opt. Express 22, 13418 (2014).

[49] R. Gordon, Limits for Superfocusing with Finite Evanescent Wave Amplification, Opt. Lett. 37, 912 (2012).

[50] Q. Cao and P. Lalanne, Negative Role of Surface Plasmons in the Transmission of Metallic Gratings with Very Narrow Slits, Phys. Rev. Lett. 88, 057403 (2002).

[51] P. B. Johnson and R. W. Christy, Optical Constants of the Noble Metals, Phys. Rev. B 6, 4370 (1972).

[52] X. Chen, H.-R. Park, M. Pelton, X. Piao, N. C. Lindquist, H. Im, Y. J. Kim, J. S. Ahn, K. J. Ahn, N. Park, D.-S. Kim, and S.-H. Oh, Atomic Layer Lithography of Wafer-Scale Nanogap Arrays for Extreme Confinement of Electromagnetic Waves, Nat. Commun. 4, 2361 (2013).

[53] H. T. Miyazaki and Y. Kurokawa, Squeezing Visible Light Waves into a 3-nm-Thick and 55-nm-Long Plasmon Cavity, Phys. Rev. Lett. 96, 097401 (2006).

[54] L. Verslegers, Z. Yu, Z. Ruan, P. B. Catrysse, and S. Fan, From Electromagnetically Induced Transparency to Superscattering with a Single Structure: A Coupled-Mode Theory for Doubly Resonant Structures, Phys. Rev. Lett. 108, 083902 (2012).

[55] M. Fernández-Suárez and A. Y. Ting, Fluorescent Probes for Super-Resolution Imaging in Living Cells, Nat. Rev. Mol. Cell Biol. 9, 929 (2008).

[56] A. Mandelis, J. Batista, and D. Shaughnessy, Infrared Photocarrier Radiometry of Semiconductors: Physical Principles, Quantitative Depth Profilometry, and Scanning Imaging of Deep Subsurface Electronic Defects, Phys. Rev. B 67, 205208 (2003). 